\title{
Association Between Obstructive Sleep Apnea and Gastroesophageal Reflux Adjusted for Obesity in Patients With Sleep Disorders: a Case-control Study
}

Shimpei Kawaguchi ( $\nabla$ shinpei.kawaguchi@ompu.ac.jp)

Osaka Medical and Pharmaceutical University: Osaka Ika Yakka Daigaku https://orcid.org/0000-00033943-4458

\section{Toshihisa Takeuchi}

Osaka Medical and Pharmaceutical University: Osaka Ika Yakka Daigaku

\section{Yuichi Kojima}

Osaka Medical and Pharmaceutical University: Osaka Ika Yakka Daigaku

\section{Kazuhiro Ota}

Osaka Medical and Pharmaceutical University: Osaka Ika Yakka Daigaku Haruhiko Ozaki

Osaka Kaisei Hospital: Osaka Kaisei Byoin

\section{Taro Iwatsubo}

Osaka Medical and Pharmaceutical University: Osaka Ika Yakka Daigaku

\section{Akitoshi Hakoda}

Osaka Medical and Pharmaceutical University: Osaka Ika Yakka Daigaku Noriaki Sugawara

Osaka Medical and Pharmaceutical University: Osaka Ika Yakka Daigaku

\section{Shinya Nishida}

Osaka Medical and Pharmaceutical University: Osaka Ika Yakka Daigaku Hironori Tanaka

Osaka Medical and Pharmaceutical University: Osaka Ika Yakka Daigaku

\section{Yosuke Mori}

Osaka Medical and Pharmaceutical University: Osaka Ika Yakka Daigaku

\section{Noriyuki Nakajima}

Osaka Medical and Pharmaceutical University: Osaka Ika Yakka Daigaku

\section{Hirohisa Tanimura}

Osaka Kaisei Hospital: Osaka Kaisei Byoin

\section{Kazuhide Higuchi}

Osaka Medical and Pharmaceutical University: Osaka Ika Yakka Daigaku 
Keywords: Obstructive sleep apnea, gastro-esophageal reflux, Obesity

Posted Date: November 2nd, 2021

DOI: https://doi.org/10.21203/rs.3.rs-1017788/v1

License: (c) (1) This work is licensed under a Creative Commons Attribution 4.0 International License. Read Full License 


\section{Abstract}

Background: The association between obstructive sleep apnea syndrome (OSAS) and gastroesophageal reflux disease has been reported in multiple studies and meta-analyses. Although obesity has been cited as a common risk factor for both conditions, in Far East Asia, sleep apnea is often attributed to craniofacial anatomy, and patients with obstructive sleep apnea are not necessarily obese. In the present study, we investigated whether there is an association between obstructive sleep apnea and gastroesophageal reflux after adjusting for obesity.

Method: The subjects were 230 patients with sleep disorders who visited the Sleep Center at Osaka Kaisei Hospital between May 2016 and November 2017. All patients underwent esophagogastroduodenoscopy. They were diagnosed with sleep disorders by a respiratory specialist, and all patients with suspected OSAS underwent polysomnography/portable monitoring. BMI was measured from the height and weight of all patients, and they were categorized into three groups: non-obese OSAS, obese OSAS, and control (non-OSAS). The presence or absence of reflux esophagitis in each group was compared using the Los Angeles classification.

Results: There were 138 patients in the obese OSAS group, 71 patients in the non-obese OSAS group, and 21 patients in the control group. There was a greater prevalence of reflux esophagitis both in the obese and non-obese OSAS groups compared with the control group (both $p<0.05$ ). Multivariate logistic regression analysis including other factors showed that alcohol consumption was the most significant factor in the non-obese OSAS group $(p<0.05)$, but the severity of OSAS as measured by the Apneahypopnea Index was not a significant factor $(p=0.64)$.

Conclusions: In patients with sleep disorders, OSAS is associated with gastroesophageal reflux regardless of obesity. Alcohol consumption is also a major factor in non-obese patients with OSAS. Since alcohol consumption is associated with the risk of esophageal squamous cell carcinoma and gastroesophageal reflux is associated with the risk of esophageal adenocarcinoma, regular upper gastrointestinal endoscopy is thought to be necessary in patients at risk.

\section{Background}

Gastroesophageal reflux (GER) is a common condition in which the retrograde flow of stomach contents into the esophagus leads to symptoms such as heartburn and acid regurgitation. Regurgitation of acidic gastric contents and biliary acid into the esophagus in gastroesophageal reflux disease (GERD) is one of the most important factors affecting the incidence of Barrett's esophagus [1]. It is also, through the development of Barrett's esophagus, associated with esophageal adenocarcinoma [2-4]. Among the United States and Europe, esophageal adenocarcinoma accounts for a high percentage of cases of esophageal cancer, and its incidence is increasing rapidly. Current guidelines recommend surveillance of individuals with known Barrett's esophagus to allow for earlier detection of esophageal adenocarcinoma, and surveillance endoscopies are recommended every 3-5 years in patients without dysplasia; if low- 
grade dysplasia is present, repeated surveillance endoscopy should be conducted within 6 months [5-7]. In Japan, although most esophageal cancers are squamous cell carcinomas, the incidence of esophageal adenocarcinoma, including cancers caused by Barrett's esophagus, has been on the rise recently.

Therefore, endoscopic surveillance is increasingly being signaled as a necessity in guidelines [8]. The most common complication of GERD is reflux esophagitis (RE), an inflammation of the mucosa of the distal esophagus that causes erosions. The Los Angeles classification, an endoscopic classification of reflux esophagitis, has also been reported to correlate with the degree of acid GER $[9,10]$, response to treatment [9], and risk of recurrence during proton pump inhibitor maintenance therapy [11].

Obstructive sleep apnea (OSA) is the most common sleep-related breathing disorder and is characterized by repetitive narrowing or collapse of the pharyngeal airway during sleep, leading to development of large negative intrathoracic pressures. Many previous studies have demonstrated a higher prevalence of GERD among patients with OSA, and a meta-analysis has shown that there is a significant correlation between the two [12]. OSA and GER share major confounding factors such as obesity, and some studies have shown a significant role of obesity, rather than OSA per se, in the relationship between OSA and GER [13]. As one of the other confounding factors, preceding arousal and shallow sleep have been reported to be related to GER [14].

However, in Far East Asia, including Japan, sleep apnea is often caused by craniofacial anatomical features such as micrognathia, and patients with OSA are not necessarily obese [15]. Therefore, we assessed the association between non-obesity related OSA and RE in patients with sleep disorders.

\section{Methods}

\section{Study population and endpoint}

Among the patients who visited the Osaka Kaisei Hospital Sleep Center between May 2016 and November 2017, 230 patients who underwent esophagogastroduodenoscopy (EGD) were included. They were diagnosed with sleep disorders by a respiratory specialist, and all patients with suspected OSAS underwent polysomnography/portable monitoring. Weight and height were measured in all patients.

Subjects were classified into the OSAS and control groups according to the following American Academy of Sleep Medicine Guidelines. Next, the OSAS group was divided into obese and non-obese patients, thus forming three groups: non-obese OSAS, obese OSAS, and sleep disorder without OSA (control group). RE was evaluated using EGD. The primary endpoint of the study was to compare the prevalence of RE in the non-obese OSAS and control groups. As secondary endpoints, factors associated with RE were examined by multivariate analysis.

\section{Polysomnography/portable monitoring}

Overnight polysomnography was conducted according to the recommendations of the American Academy of Sleep Medicine. Categorization of sleep stages and respiratory events was performed 
according to standard criteria. The severity of sleep-disordered breathing was defined by the Apneahypopnea Index (AHI) [16]. Polysomnography or portable monitoring were performed, the AHI (number of apneas and hypopneas per hour of sleep) was calculated, and OSAS was comprehensively diagnosed by a sleep disorder specialist based on this information. Non-OSAS insomnia included patients with restless legs syndrome, repetitive hypersomnia, neurosis, and psychosomatic disorders.

\section{Body mass index (BMI)}

BMI was calculated as weight in kilograms divided by height in meters squared $\left(\mathrm{kg} / \mathrm{m}^{2}\right)$. Obesity was categorized according to the World Health Organization (WHO) classification [17] and Japan Society for the Study of Obesity (JASSO) [18] as a BMI>25.0 kg/m².

\section{Esophagogastroduodenoscopy}

Following routine topical pharyngeal anesthesia with xylocaine jelly, EGD was performed in all subjects. Transoral endoscopy (EG-450WR5/H, EG-590WR, EG-590WR2, FUJIFILM, Tokyo, Japan) was used routinely at the Osaka Kaisei Hospital, whereas a transnasal endoscope (EG-530N, EG-530NW, or EGL580NW, FUJIFILM, Tokyo, Japan) was used according to patient preference. We observed the gastroesophageal junction using white light and/or blue laser imaging. We confirmed that the lower margin of the lower esophageal palisade vessels defined the gastroesophageal junction. We then diagnosed Barrett's esophagus if columnar epithelium was present on the oral side of the gastroesophageal junction [19].

In the EGD findings of all subjects, mucosal injury and RE were graded according to the Los Angeles (LA) classification [20]. For determining the RE status, grade $\mathrm{N}$ (normal findings) and grade $\mathrm{M}$ (minimal change) were added to grades $A, B, C$, and $D$ [21]. Patients graded as LA-M/N were classified into the nonreflux esophagitis (non-RE) group, while those graded as LA-A or higher were assigned to the reflux esophagitis (RE) group [22].

\section{Statistical analysis}

All data were analyzed retrospectively. Results are presented as mean \pm standard deviation. Statistical analyses were performed using JMP Pro15 (SAS Institute Inc., Cary, NC). The prevalence of RE in each group was compared using the $\chi^{2}$ test or Fisher's exact test. Multiple logistic regression analysis was used to determine the relationship between $\mathrm{AHI}$, alcohol consumption, and RE. Odds ratios (ORs) and $95 \%$ confidence intervals (Cls) were calculated to show these associations.

\section{Results}

A total of 230 patients who underwent EGD and PSG/portable monitoring were included in this study. Among the patients, 209 patients were diagnosed with OSAS, and 21 patients were in the control group and diagnosed with sleep disorders other than OSAS, such as restless legs syndrome, recurrent hypersomnia, neurosis, and psychosomatic disorder. There were 138 patients in the obese OSAS group 
who had a BMI $\geq 25 \mathrm{~kg} / \mathrm{m}^{2}$, which is considered obese according to the WHO or JASSO classification, while 71 patients in the non-obese OSAS group met the same criterion.

\section{Characteristics of the non-obese OSAS group}

There were no statistically significant differences between the non-obese OSAS and the control group in age, BMI, smoking history, hypertension, diabetes mellitus, and medication with an acid secretion inhibitor. Conversely, there were statistically significant differences in sex, alcohol consumption, and AHI between the non-obese OSAS and control groups. Male patients comprised $87.3 \%$ of the non-obese OSAS group and $47.6 \%$ of the control group $(p<0.05)$. In the non-obese OSAS group, $65.7 \%$ of patients consumed alcohol, compared to $23.8 \%$ of patients in the control group $(p<0.05)$. The AHI was $39.1 \pm 23.4 / \mathrm{h}$ in the in the non-obese OSAS group and $3.7 \pm 6.6 / \mathrm{h}$ in the control group $(\mathrm{p}<0.05)($ Table 1$)$.

\section{Characteristics of the obese OSAS group}

There were no statistically significant differences between the obese OSAS and control groups in age, smoking history, hypertension, and diabetes mellitus. Conversely, there were statistically significant differences between the obese OSAS and control group in sex, alcohol consumption, use of acid secretion inhibitor, AHI, and BMI. Male patients totaled $87.0 \%$ and $47.6 \%$ of the obese OSAS and control groups, respectively $(p<0.05)$. In the obese OSAS group, $59.9 \%$ of patients consumed alcohol, compared to $23.8 \%$ of patients in the control group $(\mathrm{p}<0.05)$. The rate of use of acid secretion inhibitors was $18.1 \%$ in the obese OSAS group and $42.9 \%$ in the control group $(p<0.05)$. The AHI was $57.9 \pm 27.6 / \mathrm{h}$ in the obese OSAS group and $3.7 \pm 6.6 / \mathrm{h}$ in the control group $(p<0.05)$. Finally, the BMI of the obese OSAS and control group was $29.5 \pm 4.0 \mathrm{~kg} / \mathrm{m}^{2}$ and $22.3 \pm 4.2 \mathrm{~kg} / \mathrm{m}^{2}$, respectively ( $<<0.05$ ) (Table 2$)$.

\section{Primary endpoint}

\section{Comparison of the prevalence of RE in non-obese OSAS and control groups}

Endoscopic findings showed that the prevalence of RE in the non-obese OSAS group (31.0\%) was significantly higher than that in the control group $(9.5 \%)(p<0.05)$.

In addition, although the difference was not significant, the prevalence of BE was higher in the non-obese OSAS group (32.4\%) than in the control group (14.3\%) $(p=0.105)$ (Table 3$)$.

\section{Secondary endpoint}

\section{Multiple logistic regression analysis of baseline covariates associated with RE risk}

Logistic regression analysis was performed on the variables with significant differences at baseline to determine the association between the AHI and alcohol consumption and RE in the non-obese OSAS 
group. The results showed that alcohol consumption was an independent risk factor for RE in the nonobese OSAS group, with an OR of $4.49(\mathrm{p}<0.01)$. On the other hand, there was no significant association between RE and the severity of SAS (AHI) $(p=0.64)$ (Table 4).

\section{Discussion}

In this cross-sectional study, we evaluated the prevalence of RE using esophagogastroduodenoscopy and found that the non-obese OSAS group (31.0\%) had a significantly higher prevalence than the non-OSAS group (control group, 9.5\%). Moreover, compared to the control group, the obese OSAS group had a higher prevalence of RE (obese OSAS vs. control: $30.4 \%$ vs. $9.5 \%$ ) and a higher prevalence of esophageal hiatal hernia (obese OSAS vs. control: $71.7 \%$ vs. $47.6 \%$ ).

Although a 2019 meta-analysis study [12] reported a strong and clear association between GERD and OSA $(H) S$, it cannot be concluded that OSAS is a direct cause of GERD. Determining the presence of RE by $\mathrm{pH} /$ impedance monitoring analyses and EGD can be useful in diagnosing GER. However, many studies have small sample sizes due to the invasive nature of these tests. On the other hand, using a noninvasive questionnaire can increase the sample size, but it is inferior to EGD as proof of GER because it may include symptoms caused by other diseases such as esophageal cancer and functional dyspepsia. Although one previous study used EGD to evaluate RE in patients with OSAS who were diagnosed by polysomnography [23], this study is, to our knowledge, the first report that has used EGD to evaluate RE in patients with non-obese OSAS, as well as the first discussion of their potential association in non-obese patients with OSAS.

\section{Association between RE and non-obese OSAS}

In a study comparing the prevalence of GER by distal esophageal pH monitoring in 63 patients with OSA and in 41 individuals without OSA diagnosed using polysomnography, patients with OSA had significantly more total reflux within 8 hours than non-OSA patients $(115$ vs. $23 ; \mathrm{P}<0.001)$ and a significantly higher percentage of patients with $\mathrm{pH}<4$ hours which used in a diagnosis of GERD $(21.4 \%$ vs. 3.7\%; $P<0.001$ ) [24]. In a Chinese study of 177 patients diagnosed with GERD and healthy controls without GERD-related symptoms or endoscopic esophagitis (97 with GERD and 80 controls) which used the Berlin Questionnaire to diagnose OSAS, significantly more patients in the GERD group had a higher risk of OSAS than those in the control group ( $36.1 \%$ vs. $17.5 \%, \mathrm{P}=0.005)$. In particular, patients with erosive reflux disease had a higher proportion of high-risk OSAS than the non-erosive reflux disease and healthy controls group ( $53.3 \%$ vs. $20.8 \%$ and $17.5 \%$, respectively, $P=0.001$ ) [25]. The only previous study that used EGD to evaluate RE in 243 patients who were divided according to polysomnography findings into OSA (98 patients) and non-OSA (145 controls) showed that endoscopy-confirmed esophagitis ( $\mathrm{p}=$ 0.027) was significantly more prevalent among patients with OSA than among those without [12]. In our study, both non-obese and obese patients with OSAS had a significantly higher prevalence of RE than sleep disordered controls without OSAS (non-obese OSAS vs. control: $31.0 \%$ vs. $9.5 \%$; $P<0.05$, obese OSAS vs. control: $30.4 \%$ vs. $9.5 \%$; $P<0.05$; Table 3$)$, This result is consistent with the results of previous 
studies mentioned above. The finding of RE confirmed by EGD is a good indicator of GER [9-11]. GER is a cause of Barrett's esophageal cancer, which is become an increasing problem and requires close surveillance [1-8]. Thus, regular surveillance of EGD is necessary not only in obese patients at risk for GERD, but also in non-obese patients with OSAS.

\section{Correlation between severity of OSA and RE in non-obese OSAS patients}

In this study, multivariate analysis showed that the $\mathrm{AHI}$, which correlates with the severity of illness in non-obese patients with OSAS, was not a significant independent risk factor for RE (Table 4). As the endinspiratory intra-esophageal pressure decreases progressively during OSA, the mechanism most likely to underlie the development of GERD in patients with OSA is decreased intrathoracic pressure due to increased inspiratory effort in the face of upper airway occlusion. However, in a study of eight patients with OSA and RE, nine OSA patients without RE, and eight healthy controls, in which polysomnography with concurrent esophageal manometry and $\mathrm{pH}$ recording were performed, it was reported that in OSA patients, GER was mainly caused by transient lower esophageal sphincter relaxation, but not by negative intra-esophageal pressure due to OSA [14]. In addition, there are many reports showing no association between GER and OSA severity $[26,27,28]$. The results of this study support these findings.

\section{Risk factors for RE in non-obese patients with OSAS}

Several studies have reported a high prevalence of RE in patients with OSAS, including some which used questionnaires to diagnose it $[23,29]$. However, many studies have reported no association between OSA severity and GER. Many studies have reported that obesity is the cause of GERD in patients with SAS considering that obesity is a common risk factor [27, 28, 30-33]. Although this study was conducted on non-obese patients with OSAS, OSA severity (AHI) was not a significant independent risk factor associated with RE, similarly to what was found in previous studies. One of the aforementioned studies, which used EGD to evaluate RE in patients with OSAS who were diagnosed by polysomnography, pointed out that esophageal hiatal hernia is a risk factor for RE [23]. However, since esophageal hiatal hernia is strongly associated with obesity [34], statistical adjustment is required if obesity and hernia are to be included as explanatory variables simultaneously in a multivariate analysis. In this study, when obese OSAS and non-obese patients with OSAS were separately compared with controls, obese patients showed a significantly greater prevalence of hiatal hernia than controls, while non-obese OSAS patients did not show a significant difference compared to controls (Table 3). Since obesity is a confounding factor, this study examined the association between OSAS and GER excluding the obesity factor by using a group non-obese patients with OSAS. Thus, the results showed that alcohol consumption was a greater risk factor than OSA severity in non-obese patients with OSAS complaining of sleep disturbances (Table 4).

The association between alcohol consumption and OSAS has been previously reported [35-37]. In a cohort of 775 males, for each increment of one drink per day, those who consumed more alcohol had $25 \%$ greater odds of sleep disordered breathing, defined as $\mathrm{AHI} \geq 5(\mathrm{OR}=1.25,95 \% \mathrm{Cl}=1.07-1.46, \mathrm{p}=$ 0.006) [38]. 
There are several possible mechanisms by which OSA is exacerbated by alcohol intake. The central nervous system depressant effects of alcohol may affect the respiratory centers that control the tone of pharyngeal muscles, thereby promoting upper airway obstruction by increasing the likelihood of pharyngeal closure during sleep. Alcohol may promote OSA by directly causing pharyngeal irritation and edema [39]. Since these mechanisms are not related to obesity and may well occur in non-obese patients, they may contribute to the risk of having RE is non-obese OSAS patients.

However, an association between alcohol consumption and GER has also been reported. Alcohol consumption may also lower esophageal sphincter pressure. In a study of 20 healthy volunteers, ingestion of white wine ( $8 \%$ alcohol) lowered the esophageal sphincter pressure. The fraction of time at $\mathrm{pH}<4$ in the first hour after ingestion of $300 \mathrm{ml}$ white wine was significantly higher than after ingesting water [40]. In a study of 24 healthy volunteers, beer (7\% alcohol) also increased the median fraction time with $\mathrm{pH}<4$ compared to water [41]. In a study of 12 healthy volunteers without GERD, the consumption of $180 \mathrm{ml}$ of vodka significantly increased reflux as evaluated by $\mathrm{pH}$ measurements [42]. In addition, basic studies in rabbits reported that exposure of the esophageal mucosa to $10 \%$ ethanol had a direct detrimental effect on the epithelium, making it more susceptible to acidic injury [43]. These reports support the possibility that alcohol is a risk factor for RE and that it is a risk factor for both OSAS and RE.

This study has several limitations. Since this was a cross-sectional study, the pathogenetic causality between OSA, alcohol consumption, and reflux esophagitis could not be determined. Moreover, the association was determined by the presence or absence of alcohol consumption, but the amount of alcohol consumed by each individual was not considered. Furthermore, as endoscopy cannot evaluate non-erosive GER and esophageal motility, comprehensive studies combined with $\mathrm{pH}$ monitoring or highresolution manometry are still necessary to gain a better understanding of GERD in patients with OSA. Moreover, we regrettably did not evaluate our patients for Helicobacter pylori infection. The inverse association between Helicobacter pylori infection and erosive esophagitis might have influenced the results of the multivariate analysis, especially with respect to RE. Finally, the study was performed in a single center. A multicenter study is needed to further validate these results.

\section{Conclusion}

This study is the first report of EGD performed in non-obese patients with OSAS to evaluate RE and highlights some clinical precautions when treating patients with OSAS. Among patients with sleep disorders, OSAS was associated with RE in both obese and non-obese patients with OSAS. In non-obese patients with OSAS, alcohol consumption was also found to be a major factor in RE. Since gastroesophageal reflux is associated with the risk of esophageal adenocarcinoma, non-obese patients with OSAS should be instructed to abstain from alcohol and undergo regular upper gastrointestinal endoscopy.

\section{Abbreviations}


OSAS, obstructive sleep apnea syndrome; GER, gastroesophageal reflux; GERD, gastroesophageal reflux disease; RE, reflux esophagitis; OSA, obstructive sleep apnea; EGD, esophagogastroduodenoscopy; WHO, World Health Organization; JASSO, Japan Society for the Study of Obesity; BE, Barrett's esophagus

\section{Declarations}

\section{Ethics approval and consent to participate}

The study was approved by the Osaka Kaisei Hospital Ethics Committee (No. 20-06). All data were fully anonymized, and informed consent was obtained via the opt-out method with approval from the ethics committee.

\section{Consent for publication}

Not applicable.

\section{Availability of data and materials}

The raw data used in this study are available from the corresponding author upon reasonable request.

\section{Competing interests}

The authors declare that they have no competing interests.

\section{Authors' contributions}

$\mathrm{KO}, \mathrm{HO}, \mathrm{TI}, \mathrm{NS}, \mathrm{SN}, \mathrm{HT}, \mathrm{YM}$, and NN contributed to the study design, analysis and interpretation of the data, and writing of the paper. TT, $\mathrm{YK}, \mathrm{AH}, \mathrm{HT}$, and $\mathrm{KH}$ contributed to the analysis of data and writing of the paper.

\section{Acknowledgements}

We give special thanks to all the staff at the Osaka Kaisei Hospital Sleep Center. We would like to thank Editage (www.editage.com) for English language editing.

\section{References}

1. Fass R, Hell RW, Garewal HS, Martinez P, Pulliam G, Wendel C et al. Correlation of oesophageal acid exposure with Barrett's oesophagus length. Gut. 2001;48:310-3

2. Richter JE, Rubenstein JH. Presentation and epidemiology of gastroesophageal reflux disease. Gastroenterology. 2018;154(2):267-76

3. Eusebi LH, Cirota GG, Zagari RM, Ford AC. Global prevalence of Barrett's oesophagus and oesophageal cancer in individuals with gastro-oesophageal reflux: a systematic review and metaanalysis. Gut. 2021;70(3):456-63 
4. Lagergren J, Bergström R, Lindgren A, Nyrén O. Symptomatic gastroesophageal reflux as a risk factor for esophageal adenocarcinoma. N Engl J Med. 1999;340(11):825-31

5. American Gastroenterological Association, Spechler SJ, Sharma P, Souza RF, Inadomi JM, Shaheen NJ. American Gastroenterological Association medical position statement on the management of Barrett's esophagus. Gastroenterology. 2011;140(3):1084-91

6. Fitzgerald RC, di Pietro M, Ragunath K, Ang Y, Kang JY, Watson P, et al. British Society of Gastroenterology guidelines on the diagnosis and management of Barrett's oesophagus. Gut. 2014;63(1):7-42

7. Spechler SJ. Barrett esophagus and risk of esophageal cancer: a clinical review. JAMA. 2013;310(6):627-36

8. Matsuhashi N, Sakai E, Ohata K, Ishimura N, Fujisaki J, Shimizu T, et al. Surveillance of patients with long-segment Barrett's esophagus: A multicenter prospective cohort study in Japan. J Gastroenterol Hepatol. 2017;32(2):409-14

9. Lundell LR, Dent J, Bennett JR, Blum AL, Armstrong D, Galmiche JP, et al. Endoscopic assessment of oesophagitis: clinical and functional correlates and further validation of the Los Angeles classification. Gut. 1999;45:172-80

10. Ghoshal UC, Chourasia D, Tripathi S, Misra A, Singh K. Relationship of severity of gastroesophageal reflux disease with gastric acid secretory profile and esophageal acid exposure during nocturnal acid breakthrough: a study using 24-h dual-channel pH-metry. Scand J Gastroenterol. 2008;43(6):654-61

11. Fujimoto K, Hongo M, Maintenance Study Group. Risk factors for relapse of erosive GERD during long-term maintenance treatment with proton pump inhibitor: a prospective multicenter study in. Japan. J Gastroenterol. 2010;45:1193-200

12. Wu ZH, Yang XP, Niu X, Xiao XY, Chen $X$. The relationship between obstructive sleep apnea hypopnea syndrome and gastroesophageal reflux disease: a meta-analysis. Sleep Breath. 2019;23(2):389-97

13. Shepherd K, Orr W. Mechanism of gastroesophageal reflux in obstructive sleep apnea: airway obstruction or obesity? J Clin Sleep Med. 2016;12(1):87-94

14. Kuribayashi S, Kusano M, Kawamura O, Shimoyama Y, Maeda M, Hisada T, et al. Mechanism of gastroesophageal reflux in patients with obstructive sleep apnea syndrome. Neurogastroenterol Motil. 2010;22(6):611-e172

15. Li KK, Kushida C, Powell NB, Riley RW, Guilleminault C. Obstructive sleep apnea syndrome: a comparison between Far-East Asian and white men. Laryngoscope. 2000;110(10 Pt. 1):1689-93

16. American Academy of Sleep Medicine. International classification of sleep disorders. 3rd ed. Darien, IL: American Academy of Sleep Medicine; 2014

17. WHO Expert Consultation. Appropriate body-mass index for Asian populations and its implications for policy and intervention strategies. Lancet. 2004;10:363(9403):157-63

18. Examination Committee of Criteria for 'Obesity Disease' in Japan; Japan Society for the Study of Obesity. New criteria for "obesity disease" in. Jpn Circ J. 2002;66(11):987-92 
19. the Japan Esophageal Society. Japanese classification of esophageal cancer. 11th ed.: 1. Esophagus; 2017;14(1). p. 1-36

20. Hoshihara Y, Hashimoto M. Endoscopic classification of reflux esophagitis (in Japanese with English abstract). Nihon Rinsho. 2000;58:1808-12

21. Hongo M. Minimal changes in reflux esophagitis: red ones and white ones. J Gastroenterol. 2006;41:95-9

22. Armstrong D, Bennett JR, Blum AL, Dent J, De Dombal FT, Galmiche JP et al. The endoscopic assessment of esophagitis: a progress report on observer agreement. Gastroenterology. 1996;111:85-92

23. Zenda T, Hamazaki K, Oka R, Hagishita T, Miyamoto S, Shimizu J et al. Endoscopic assessment of reflux esophagitis concurrent with hiatal hernia in male Japanese patients with obstructive sleep apnea. Scand J Gastroenterol. 2014;49(9):1035-43

24. Ing AJ, Ngu MC, Breslin ABX. Obstructive sleep apnea and gastroesophageal reflux. Am J Med. 2000;108:120-5. doi:10.1016/S0002-9343(99)00350-2

25. Chen Y, Xiong L, Zeng J, Wei YG, Tan Y. Gastroesophageal reflux disease is associated with high risk of obstructive sleep apnea syndrome. Zhonghua Nei Ke Za Zhi. 2018;57(11):824-9

26. Shepherd K, Hillman D, Holloway R, Eastwood P. Mechanisms of nocturnal gastroesophageal reflux events in obstructive sleep apnea. Sleep Breath. 2011;15(3):561-70

27. Kim HN, Vorona RD, Winn MP, Doviak M, Johnson DA, Ware JC. Symptoms of gastro-oesophageal reflux disease and the severity of obstructive sleep apnoea syndrome are not related in sleep disorders center patients. Aliment Pharmacol Ther. 2005;21(9):1127-33

28. Hawryłkiewicz I, Pływaczewski R, Dziedzic D, Czerniawska J, Górecka D. Gastroesophageal reflux disease (GERD) in patients with obstructive sleep apnoea syndrome (OSAS). Pneumonal Alergol Pol. 2006;74(4):361-4

29. You CR, Oh JH, Seo M, Lee HY, Joo H, Jung SH et al. Association between Non-erosive Reflux Disease and high risk of Obstructive Sleep Apnea in Korean Population. J Neurogastroenterol Motil. 2014;20(2):197-204

30. Basoglu OzenK, Vardar R, Tasbakan MS, Ucar ZZ, Ayik S, Kose T et al. Obstructive sleep apnea syndrome and gastroesophageal reflux disease: the importance of obesity and gender. Sleep Breath. 2015;19(2):585-92

31. Erdem D, Yılmaz YF, Özcan M, Titiz A, Özlügedik S, Ünal A. Correlation of sleep-disordered breathing and laryngopharyngeal reflux: a two-channel triple-sensor pHmetry catheter study. Eur Arch Otorhinolaryngol. 2018;275(10):2585-92

32. Noronha AC, de Bruin VM, Nobre e Souza MA, de Freitas MR, Araújo Rde P, Mota RM et al. Gastroesophageal reflux and obstructive sleep apnea in childhood. Int J Pediatr Otorhinolaryngol. 2009;73(3):383-9

33. Oztürk O, Oztürk L, Ozdogan A, Oktem F, Pelin Z. Variables affecting the occurrence of gastroesophageal reflux in obstructive sleep apnea patients. Eur Arch Otorhinolaryngol. 
2004;261(4):229-32

34. Nocon M, Labenz J, Willich SN. Lifestyle factors and symptoms of gastro-oesophageal reflux - a population-based study. Aliment Pharmacol Ther. 2006;23(1):169-74

35. Baik I, Seo HS, Yoon D, Kim SH, Shin C. Associations of sleep apnea, NRG1 polymorphisms, alcohol consumption, and cerebral white matter hyperintensities: analysis with genome-wide association data. Sleep. 2015;38(7):1137-43

36. Koyama RG, Esteves AM, Oliveira e Silva L, Lira FS, Bittencourt LR, Tufik S. Lia R, Marco Tulio Mello. Prevalence of and risk factors for obstructive sleep apnea syndrome in Brazilian railroad workers. Sleep Med. 2012;13(8):1028-32

37. Gothe B, Strohl KP, Levin S, Cherniack NS. Nicotine: a different approach to treatment of obstructive sleep apnea. Chest. 1985;87(1):11-7

38. Peppard PE, Austin D, Brown RL. Association of alcohol consumption and sleep disordered breathing in men and women. J Clin Sleep Med. 2007;3(3):265-70

39. Scrima L, Broudy M, Nay KN, Cohn MA. Increased severity of obstructive sleep apnea after bedtime alcohol ingestion: diagnostic potential and proposed mechanism of action. Sleep. 1982;5(4):318-28

40. Pehl C, Pfeiffer A, Wendl B, Kaess H. Different effects of white and red wine on lower esophageal sphincter pressure and gastroesophageal reflux. Scand J Gastroenterol. 1998;33(2):118-22

41. Pehl C, Wendl B, Pfeiffer A, Schmidt T, Kaess H. Low-proof alcoholic beverages and gastroesophageal reflux. Dig Dis Sci. 1993;38(1):93-6

42. Kaufman SE, Kaye MD. Induction of gastro-oesophageal reflux by alcohol. Gut. 1978;19(4):336-8

43. Bor S, Bor-Caymaz C, Tobey NA, Abdulnour-Nakhoul S, Orlando RC. Esophageal exposure to ethanol increases risk of acid damage in rabbit esophagus. Dig Dis Sci. 1999;44(2):290-300

\section{Tables}

Due to technical limitations, table 1 to 4 PDFs are only available as a download in the Supplemental Files section.

\section{Figures}




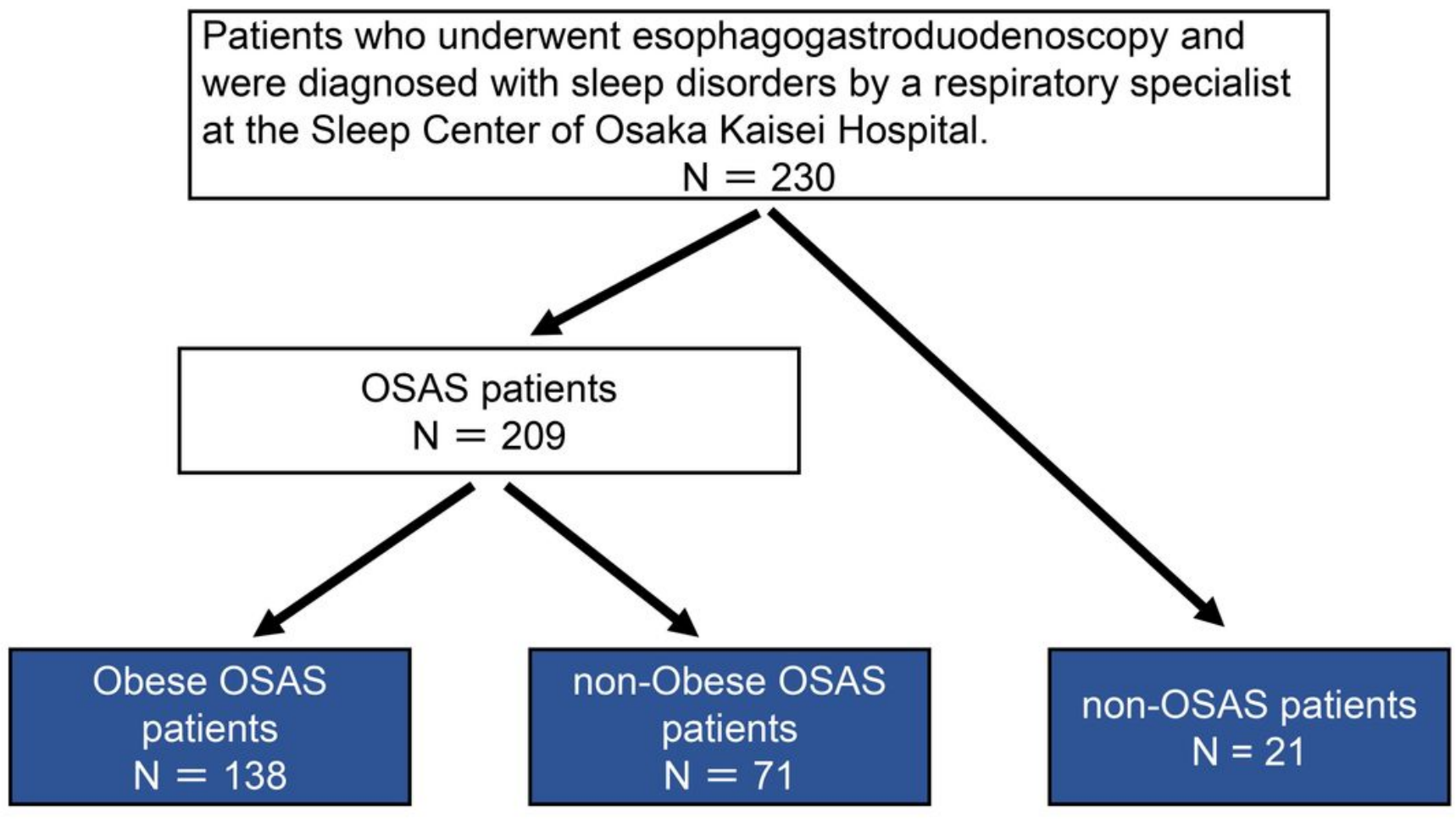

Figure 1

Study flowchart. OSAS: Obstructive sleep apnea syndrome

\section{Supplementary Files}

This is a list of supplementary files associated with this preprint. Click to download.

- Table1.pdf

- Table2.pdf

- Table3.pdf

- Table4.pdf 